\title{
Brussels Studies
}

La revue scientifique électronique pour les recherches sur Bruxelles / Het elektronisch wetenschappelijk tijdschrift voor onderzoek over Brussel / The e-journal for academic research on Brussels

Collection générale | 2014

\section{Un RER à Bruxelles ? Socio-histoire des rivalités et des régulations politiques (1989-2013)}

A RER in Brussels? A sociological history of rivalries and political regulations (1989-2013)

Een GEN in Brussel? Socio-historisch onderzoek naar de rivaliteiten en politieke reguleringen (1989-2013)

\section{Ludivine Damay}

\section{(2) OpenEdition}

\section{Journals}

Édition électronique

URL : http://journals.openedition.org/brussels/1205

DOI : 10.4000/brussels. 1205

ISSN : 2031-0293

Éditeur

Université Saint-Louis Bruxelles

Référence électronique

Ludivine Damay, «Un RER à Bruxelles ? Socio-histoire des rivalités et des régulations

politiques (1989-2013) », Brussels Studies [En ligne], Collection générale, $n^{\circ} 74$, mis en ligne le 17 février 2014, consulté le 30 avril 2019. URL : http://journals.openedition.org/brussels/1205 ; DOI : 10.4000/ brussels. 1205

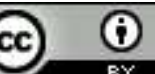




\title{
BRUSSELS STUDIES
}

Numéro 74, 17 février 2014. ISSN 2031-0293

\author{
Ludivine Damay
}

\section{Un RER à Bruxelles ? Socio-histoire des rivalités et des régulations politiques (1989-2013)}

Cet article retrace l'historique du projet de Réseau Express Régional (RER) de, vers, dans et autour de Bruxelles en mettant en avant, d'une part, les grands conflits entre les différents acteurs qui ont sous-tendu le dossier dès l'origine. Le RER est en effet au cœur de rivalités autour des usages et des usagers qu'il devrait privilégier, des visions de la ville qu'il favoriserait ainsi que des politiques de mobilité concrète à développer, du rôle de la Société Nationale des Chemins de fer Belges (SNCB) à cet égard. D'autre part, l'article retrace également les différentes étapes de la régulation politique du dossier, relevant les phases d'avancées et de blocages qui jalonnent l'histoire du RER. En éclairant l'histoire de ces rivalités et de ces antagonismes qui n'ont jamais été vidés de leur substance, cet article donnera une meilleure vue de la situation actuelle du dossier, illustrant au passage la force opératoire des quiproquos.

Ludivine Damay est docteure en sciences politiques et sociales. Elle est actuellement chercheure et chargée de cours à l'Université Saint-Louis - Bruxelles, ainsi qu'à l'Université Catholique de Louvain. Sur le même sujet, elle a déjà publié un article dans la revue Flux, n91, janvier-mars 2013 et obtenu un prix pour une communication lors du colloque organisé par l'Institut pour la ville en mouvement, à Paris, en mars 2012. Ses recherches portent sur l'action publique, la participation citoyenne ainsi que le développement urbain et régional. 


\section{Introduction ${ }^{1}$}

1. La Région de Bruxelles-Capitale (RBC) est régulièrement présentée sous les traits d'une Ville-Région au bord de l'asphyxie sous la pression automobile. Depuis la création de la RBC en 1989, cette problématique est au centre des débats politiques et sociétaux. La problématique de la mobilité à Bruxelles est complexe pour plusieurs raisons. La Région est d'abord confrontée à une politique du « tout à l'automobile » [Hubert, 2008] bien antérieure à sa création, qui a, dès la fin des années 1950, profondément marqué l'espace urbain en accélérant la périurbanisation. Cet étalement urbain et l'attraction de la capitale en termes d'emploi ont pour conséquence un taux de navetteurs quotidiens de longue distance particulièrement important au point qu'on évoque une "société de navetteurs » [Montulet, et al., 2008]. Le phénomène de la navette - d'abord ferroviaire, puis autoroutière - a été favorisé par les pouvoirs publics et incarne " un élément constitutif de l'urbanisation de la Belgique et de Bruxelles en particulier. " [Dessouroux, 2008, p. 4] Le problème de mobilité à Bruxelles est également confronté au fait que la Région, au même titre que la Wallonie et la Flandre, est certes compétente sur son territoire en matière de transport public mais d'autres niveaux de pouvoir jouent également des rôles substantiels en la matière. Le niveau fédéral pilote la Société nationale des chemins de fer belges (SNCB), le niveau communal gère certaines voiries et adopte un plan communal de mobilité. L'éparpillement des compétences [Aussems, 2009] rend complexe la gestion de la mobilité d'autant plus qu'à l'empilement des niveaux de pouvoirs, il faut ajouter l'échelle des problèmes à prendre en compte qui ne correspond que rarement aux limites institutionnelles des territoires, ainsi que la complexité d'un phénomène qui surpasse la seule question des déplacements. Résoudre la mobilité à Bruxelles implique de facto de dépasser les limites territoriales de la Région, les limites institutionnelles des compétences régionales et les cloisonnements sectoriels.
2. Dans ce contexte, l'idée de créer un Réseau Express Régional (RER) de, vers, dans et autour de Bruxelles s'est diffusée peu à peu. Dans le cadre de cet article, nous retracerons quelques grands enjeux autour du RER en mettant en lumière comment certains acteurs ont façonné ceux-ci. La perspective socio-historique permet en effet d'apprécier la genèse et la profondeur des phénomènes sociaux, la manière dont ceux-ci ont été construits et mis à l'agenda comme " problème public » [Noiriel, 2006 ; Damay et al, 2011]. Nous montrerons ainsi que le RER est, dès le début du projet, au cœur de nombreux conflits d'usage, conflits autour de visions de la ville mais aussi de conflits à propos des politiques concrètes de mobilité à mettre en oeuvre. Ces conflits pèsent fortement sur l'espace de régulation politique autour du dossier RER, régulation que nous aborderons dans un second temps. Des formes de gouvernance négociées ont en effet été mises en place en vue de construire ce " nouveau » réseau de transport en commun, néanmoins certains blocages semblent inextricables. Dans l'impasse politique et budgétaire, le projet RER se voit même devancé voire concurrencé par d'autres projets alternatifs de réseaux ferrés récemment portés dans l'espace public.

\section{Le RER : des rivalités complexes}

3. Des projets précurseurs ont existé avant celui du RER, tel le projet de métropolitain, dont il était question dans les années 1920 et 1930 [Van Meerteen, et al., 2002, p. 32]. Dès 1968, l'avant-projet de plan de secteur de 1976 prévoit aussi une ébauche de RER en complément du métro [Tellier, 2012, p. 185]. On note également des revendications locales (ou au contraire des craintes) de mise à trois voies de lignes ferrées afin de mieux desservir certaines communes bruxelloises au milieu des années 1970². Cela dit, les acteurs principaux du dossier s'accordent à dire que c'est véritablement vers la fin des années 1980

${ }^{1}$ Cet article a été rédigé dans le cadre d'un projet de recherche "Prospective Research for Brussels ", financé par la Région de Bruxelles-Capitale (RBC) entre janvier 2011 et juin 2013. L'étude est centrée sur les acteurs bruxellois du dossier RER même si les autres perspectives ne sont pas évincées. Les résultats reproduits ici sont basés sur une analyse documentaire (compte-rendu de débats parlementaires, articles de presse, mémorandum d'associations, rapports de bureaux d'études, rapports du comité de pilotage RER, etc.) et sur la réalisation d'entretiens (20) semi-directifs avec des acteurs clés du dossier. Nous souhaitons remercier les lecteurs anonymes de ce papier pour leurs commentaires sur le projet, ainsi que Kevin Lebrun, Michel Hubert et Benjamin Wayens.

2 HENROTIN, A., « Le plan star 21 inquiète la population proche de la Ligne 161. Le spectre du RER hante Boitsfort. », Le Soir, le 10 mai 1991 , p. 22. 
que le projet entre dans le débat public sous l'effet conjugue de deux événements. Tout d'abord, en décembre 1988, Stratec (un bureau d'étude indépendant, créé en 1984) dépose son rapport final à la SNCB, commanditaire d'une étude cherchant à améliorer la part de marché de l'entreprise vers Bruxelles. Les conclusions de l'étude sont sans appel : la part de marché de la SNCB diminue dans un rayon de $37,5 \mathrm{~km}$ autour de Bruxelles alors que les plus hauts potentiels se situent dans cette couronne qui constitue un important vivier de navetteurs. Suite à cela, les auteurs invitent la SNCB à « remplacer partiellement l'offre actuelle de type inter-urbain par une offre de type sub-urbain $[\ldots] ;[\ldots]$ sur son trajet urbain ce type de service drainerait également une partie des déplacements intra-urbains et périphériques [...] et apporterait ainsi une contribution positive à l'amélioration du niveau des transports en commun urbains de Bruxelles $»^{3}$. Si le rapport ne mentionne pas le sigle "RER », il en dessine les objectifs qui répondent à une intention commerciale de la SNCB, en perte de vitesse à l'époque. La SNCB embraie sur cette thématique et un plan plus précis d'un futur réseau suburbain, à l'image du RER parisien, comme elle l'affirme ${ }^{4}$, s'ébauche. Le plan Star 21, présenté par la SNCB en 1989, annonce également la densification "d'un réseau suburbain » assurant une " desserte fréquente de Bruxelles ». ${ }^{5}$ L'entreprise publique reculera cependant, évoquant à maintes reprises des problèmes techniques et financiers. Pourtant, deuxième événement, le RER s'invite explicitement ailleurs, dans la première campagne électorale au niveau de la RBC, en juin 1989. Plusieurs partis se réfèrent à ce réseau, avec en toile de fond, les thématiques de la congestion automobile. "L'asphyxie de Bruxelles » est une thématique qui prend de l'importance dans les discours politiques, relayée par les médias qui lui donnent aussi une vision catastrophiste. Dès la mise en place de la Région, on évoque la néces sité d'un plan de déplacement pour Bruxelles (le futur plan IRIS) qui ferait la part belle aux transports en commun, dont le train. De nom- breux acteurs bruxellois relèvent d'ailleurs le volontarisme politique du ministre des Travaux Publics et des Communications, Jean-Louis Thys, et de son cabinet, dans ce dossier RER. Cela dit, du côté de l'opposition écologiste au premier exécutif régional bruxellois, on relève déjà que malgré les discours: "il y a des consensus - dont celui à propos de la voiture - qui sont difficiles à briser ${ }^{6}$. La place de la voiture en ville est un des grands éléments qui façonnent l'espace des rivalités, dès cette époque, mais il n'est pas le seul. Nous présentons ici trois grands débats qui structurent et jalonnent l'existence et l'avancée du projet RER.

\subsection{Rivalités d'usages}

4. A qui servirait ce RER ? Quels types d'usages privilégierait-il ? Quels seraient ses effets sur la mobilité mais aussi sur les stratégies résidentielles des ménages ainsi que celles des acteurs économiques ? De houleux débats traitent de ces questions dès l'entame du projet. Si ce sont vraiment les politiques bruxellois qui mènent la danse pour promouvoir le RER en prenant certaines initiatives en matière de négociation ou de publicisation de l'enjeu, leur position est cependant loin d'être unanime.

5. Pour certains politiques, le RER doit certes répondre au phénomène de la navette automobile en provoquant un report modal de la voiture vers le train, mais il doit également régler le problème de mobilité interne à Bruxelles en servant les usagers bruxellois. Or, l'exécutif bruxellois critique les visées de la SNCB qui « ne prend aucunement en compte la mobilité interne à Bruxelles.» ${ }^{7}$ En plus de la desserte existante, l'exécutif bruxellois suggère de promouvoir deux lignes, vers l'ouest et l'est de Bruxelles comme préfiguration du RER et qui soutiendraient le développement d'une mobilité interne en permettant une meilleure accessibilité de zones excentrées, moins bien desservies par le réseau ferré actuel qui passe majoritairement dans la jonction Nord-

\footnotetext{
3 STRATEC, Etude de la desserte ferroviaire de Bruxelles et environ, rapport final pour la SNCB, 5 décembre 1988, p. 31.

4 VANTROYEN, J-C. "Le chemin de fer pour éviter l'asphyxie de la capitale ", Le Soir, 29 mai 1989.

${ }^{5}$ DEPAS, G., «Star 21 : répondre au 21 ème siècle (détails du projet de la SNCB) ", Le Soir, 13 novembre 1989, p. 4

${ }^{6}$ Conseil de la RBC, Compte rendu intégral, Séance plénière du jeudi 13 juillet 1989, p. 44

7 ALSTEENS, O., « Le plan Star 21 de Mr Dehaene a oublié les Bruxellois », Le Soir, 21 avril 1990, p. 10.
} 
Midi. La promotion de cette mobilité interne à Bruxelles n'est pas forcément du goût des autres Régions d'abord parce qu'elles cherchent, de leur côté, à favoriser une desserte la plus rapide possible au centre de Bruxelles pour leurs navetteurs, ensuite parce qu'elles y voient une forme de hold-up sur les financements fédéraux. La Flandre, par exemple, s'exprime en 1992, par le biais de son Ministre des transports : «Si nous analysons la situation actuelle, nous avons plutôt l'impression que la Région de Bruxelles-Capitale souhaite résoudre ses problèmes par l'engagement des autres et par des ponctions dans les coffres au niveau fédéral, au niveau de la Région flamande ou de la Région wallonne ${ }^{8}$. II est évident que pour les autres Régions, tout comme pour la SNCB qui y voit son intérêt en termes de parts de marché, ce sont bien les navetteurs qui sont les premiers usagers potentiels. Paradoxalement peut-être, certains politiques bruxellois sont bien de cet avis : Ie RER n'est pas fondamentalement une affaire de Bruxellois ! « Ce sont les Flamands et les Wallons qui habitent dans un rayon de 30 kilomètres autour de Bruxelles qui ont besoin d'un Réseau express régional. Les Bruxellois, eux, n'en seront que les utilisateurs occasionnels. Nulle raison donc que la Région bruxelloise prenne principalement en charge la construction d'un réseau qui risque d'accentuer encore l'exode de ses habitants " ${ }^{9}$. L'objectif visé est surtout d'éviter de participer lourdement à son potentiel financement.

6. Dans la citation précédente, se profile un autre élément du débat : et si le RER servait surtout à vider Bruxelles de ses habitants ? Pour certains, le RER risque en effet d'accentuer l'exode urbain des ménages riches en dehors de Bruxelles en offrant un moyen rapide et fréquent d'atteindre les centres d'emplois bruxellois tout en offrant aux ex-citadins une qualité de vie supposée plus agréable dans les " campagnes " wallonnes ou flamandes et un marché immobilier lui aussi plus attractif. C'est un débat somme toute classique, celui de l'étalement urbain. La crainte de voir se précipiter les Bruxellois en dehors de Bruxelles grâce à ce réseau est présent dès l'entame du projet ${ }^{10}$. Le
RER est vu comme un service intéressant mais déstabilisateur, incitant potentiellement à de nouveaux départs. Cet argument trouve des appuis dans le contexte démographique bruxellois (qui a fort changé depuis) : entre 1971 et 1991 la RBC a perdu 11 pourcents de sa population alors que la périphérie en a gagné 20. Le RER est ainsi décrit comme la pire chose pour une Région qui est aussi sous-financée notamment en raison d'un impôt prélevé au lieu de résidence. L'Atelier de Recherche et d'Action Urbaines (I'ARAU) a été et continue à être particulièrement critique par rapport aux effets du RER pour Bruxelles et " l'habitabilité urbaine » [Schoonbrodt, 2007]. Des études modélisant les effets du RER sur la localisation des ménages confirmeront, plus tard, ce risque d'exode [Boon et Gayda, 2000]. Certains politiques bruxellois sont ainsi échaudés par ce qui pourrait s'apparenter à la transformation de la «[...] capitale en un espace d'usage qui relègue au second plan les attentes légitimes des habitants et la qualité de leur cadre de vie ${ }^{11}$. Le projet de RER pour contrer cet exode doit s'attaquer aussi à la suprématie de la voiture, notamment par des mesures d'accompagnement. Nous y reviendrons.

\subsection{Rivalités à propos des visions de la ville}

7. Derrière ces positions qui privilégient tantôt les Bruxellois, tantôt les navetteurs, on voit également apparaître, tout au long de la socio-histoire du RER, différentes visions de la ville ou différents référentiels qui guident les positions de chacun. Bruxelles est-elle une ville hypercentrée, une ville de bureaux concentrés autour des principales gares de la jonction NordMidi et du quartier européen ? Bruxelles est-elle au contraire une ville qui doit revenir à ses habitants qui ont déjà payé un lourd tribut au développement ferroviaire? Ces images différenciées de la ville se retrouvent dans les propositions concrètes qui préfigurent l'offre du futur RER. Si on caricature (à peine) le débat, les Wallons et les Flamands veulent accéder le plus vite possible au centre de Bruxelles, par la Jonction Nord-Midi ou par le quartier européen. Autant les points d'arrêt doivent être nombreux sur leur territoire respectif, autant ils doivent être limités sur le territoire

${ }^{8}$ SAUWENS, J., Ministre communautaire des Transports, du Commerce extérieur et de la Réforme de l'Etat, Le RER à toute vitesse, Actes de la table ronde du 5 juin 1992 , Bruxelles, p. 5.

${ }^{9}$ TELLIER, D., "Le SP appuie le plan Transport SNCB. Le RER n'est pas l'affaire des Bruxellois ", Le Soir, $1^{\text {er }}$ août 1997, p. 4.

${ }_{10}$ Conseil de la Région de Bruxelles-Capitale, Bulletin des interpellations et des questions orales et d'actualité, 13 mars 1992, p. 197.

${ }^{11}$ Charles Picqué, Pour Bruxelles. Entre périls et espoirs, Bruxelles, Editions Racine, 1999, p. 123. 
bruxellois puisqu'il s'agit de ne pas alourdir le temps de parcours en arrêts considérés comme inutiles [Frenay, 2009]. Pas question non plus de faire circuler des trains vers des pôles de moindre importance, comme la gare de l'Ouest. Bruxelles est ainsi limitée à son extrême centre et au quartier européen, c'est une ville de navetteurs avec quelques pôles d'emplois. En revanche, la RBC cherche à maximiser le nombre d'arrêts sur son territoire et à favoriser l'utilisation plus harmonieuse d'un réseau ferré existant pour la mobilité interne à Bruxelles. Elle désencombrerait ainsi son réseau de métro, lui aussi saturé sur ses parties centrales. Cela dit, là encore, les positions sont plus complexes qu'il n'y paraît. Par exemple, le plan Régional de Développement de 1995, au travers de sa politique $\mathrm{ABC}$, vise à positionner les activités tertiaires sur « les principaux nœuds de transports en commun " existants (Nord, Midi, Arts-Loi, Schuman,...) [Hubert, et al. 2008 ; de Keersmaecker, 2005]. De nombreuses craintes jaillissent aussi à Bruxelles, mais pas seulement, quant au potentiel destructeur des infrastructures du RER, et notamment la mise à quatre voies, sur le tissu urbain existant. Certaines communes et certains collectifs (des associations autour d'une cause comme la mobilité alternative ou encore des regroupements de riverains) freinent donc l'avancée du projet ${ }^{12}$ ou s'interrogent eux aussi sur l'opportunité de relier leur territoire à l'ouest de Bruxelles, plutôt qu'au centre ${ }^{13}$.

8. De manière beaucoup plus récente, l'image de Bruxelles est davantage façonnée comme ville " polycentrique ", surtout dans les débats politiques concernant le dernier plan de développement de Bruxelles, le Plan Régional de Développement Durable, dont le projet est approuvé en première lecture par le Gouvernement bruxellois le 26 septembre 2013. Cette polycentricité s'appuie sur le fait que le centre n'est pas forcément l'origine ni l'arrivée des voyages effectués en ville et qu'il faut donc densi- fier et favoriser la mixité urbaine autour des nœuds intermodaux. Cela dit, certaines analyses critiquent l'usage uniquement rhétorique de ce terme sans que des mesures concrètes ne viennent encore le mettre en pratique [Casabella et Frenay, 2009].

9. D'autres images de la ville sont également en concurrence du point de vue des référentiels utilisés dans les espaces de négociation ou dans les débats publics. Le RER peut ainsi contribuer à rendre « respirable » la ville, à développer une mobilité " durable ", comme "mode de transport le plus respectueux de l'environnement ${ }^{14}$. Le RER devrait favoriser ce report modal de la voiture vers des transports alternatifs, report annoncé comme objectif politique dans les plans de mobilité IRIS 1 et IRIS 2 de la RBC. Les associations environnementales des trois Régions sont d'ailleurs globalement favorables à ce réseau ${ }^{15}$ moyennant la mise en œuvre de mesures d'accompagnement, de la diminution des nuisances pour les riverains et, pour les Bruxellois, de la prise en compte de la mobilité interne à Bruxelles. A côté de l'image de Bruxelles comme " écocapitale » [IRIS 2, p. 18], le RER, grâce au report modal engendré, pourrait également permettre d'assurer une meilleure accessibilité aux pôles économiques et aux entreprises. Le moindre des paradoxes n'est pas, en effet, que le RER en réduisant la pression automobile permettrait.. aux véhicules restants sur les routes de mieux circuler, ce qui est un argument important pour certains acteurs comme les différents partenaires sociaux des trois Régions l'ont exprimé ${ }^{16}$. II en va ainsi de Bruxelles comme « capitale économique ».

\subsection{Rivalités autour des politiques de mobilite}

10. Le RER suscite également des controverses socio-techniques sur les politiques concrètes à mettre en œuvre, leur degré de faisabilité

\footnotetext{
(http://www.marccools.be/region.html, consulté le 23/12/2013).

${ }^{14}$ BOVY, L., "RER : un projet de mobilité nécessaire ", Présentation au Conseil économique et social, 28 octobre 2010.

${ }^{15}$ Voir par exemple : Inter-environnement Wallonie, avis d'IEW sur le RER, $1^{\text {er }}$ septembre 2004.

16 Déclaration commune des SERV, CESRBC et CESRW, Le Réseau Express Régional, 27 juin 2006.
}

12 SCHOUNE, C., "Uccle-Stalle réaiguille les plans du RER », Le Soir, 10 mai 2000 et http://www.uccle.be/fr/services-communaux/urbanisme/rer-1 (consulté le 23/12/2013).

13 « La SNCB vient de présenter un schéma provisoire d'exploitation du RER. Si celui-ci va améliorer l'accès des navetteurs à Bruxelles, il ne va par contre pas favoriser les déplacements de Bruxelles à Bruxelles en train. C'est ainsi qu'à Uccle, la ligne 124 (qui dessert les gares de Linkebeek, de Calevoet et de Stalle) n'ira plus à la gare du Midi mais à Molenbeek à la gare de l'Ouest. Même si le souci d'alléger la jonction nord-midi est compréhensible, c'est, pour l'Echevin des Travaux et de la Mobilité d'Uccle Marc Cools, inacceptable 
leurs effets potentiels. Le moins que l'on puisse dire est que, par rapport à ces enjeux, la SNCB-holding a la main dans la mesure où elle est propriétaire de l'infrastructure ferroviaire (via sa filiale Infrabel), maitre d'œuvre des travaux qui touchent son réseau et exploitant du trafic ferroviaire (via l'opérateur SNCB). La situation monopolistique de l'entreprise publique dans la régulation du rail a souvent été décriée : c'est elle qui a été pointée du doigt dans le blocage du projet à maintes reprises. La presse signale d'ailleurs que, selon la SNCB et dès l'entame du projet, « il serait impossible d'utiliser l'infrastructure existante. Pour des raisons d'incompatibilité de signalisation, d'alimentation ou de circulation " 17 . Selon son propre aveu, le RER n'est pas son " core business ", qui serait davantage celui des entreprises régionales de transports. En tant qu'entreprise nationale, elle a moins d'intérêt pour le développement ferré sub-urbain et privilégie son plan IC/IR (entre villes et régions) et son développement international. Autres impératifs avancés dans le débat par la SNCB et qui ont un impact sur les politiques menées : la sécurité du transport ; la robustesse du réseau ; la rentabilité et la vitesse commerciale. La suspicion règne d'ailleurs sur ses actions : si la SNCB a accepté le RER, c'est parce que ce projet lui permettait d'augmenter ses capacités en termes d'infrastructures (les mises à quatre voies, le tunnel Schuman-Josaphat) au profit de la robustesse de son réseau ${ }^{18}$.

11. Mais le débat sur les politiques de mobilité à mener ne se limite pas aux infrastructures à construire et au schéma d'exploitation du RER. Plusieurs études indiquent en effet que le RER ne résoudra pas les problèmes de mobilité si des mesures drastiques d'accompagnement ne sont pas prises, mesures visant, d'une part, à rendre plus attractif le train en pénalisant l'usage de la voiture de différentes façons (comme la politique de stationnement, la réduction des voies de circulation ou la mise en place d'un péage urbain) et, d'autre part, à jouer sur les demandes de mobilité via une politique d'aménagement du territoire privilégiant la den- sité et le développement urbain autour des pôles mieux desservis en transport en commun. Les mesures d'accompagnement ont partie liée avec l'exode et l'étalement urbains. Outre la première étude déjà mentionnée [Boon et Gayda, 2000] menée de 1996 à 1998, d'autres études plus tardives, en 2002 et 2003, indiquent d'ailleurs dans quelles proportions l'exode urbain sera facilité si on ne prend pas certaines mesures d'accompagnement ${ }^{19}$. Sans surprise, d'après ces études, la seule augmentation de l'offre de transport en commun ne suffira pas à enrayer l'exode urbain : il faut s'attaquer à la suprématie de la voiture, via des mécanismes de péage urbain et de restrictions de stationnement ${ }^{20}$. La RBC va batailler pour imposer aux autres acteurs ces mesures d'accompagnement qu'elle juge davantage nécessaire que les autres Régions. Pourtant, il ne suffit pas que les autres Régions construisent des parkings autour des futures gares RER, la RBC et les 19 communes qui la composent doivent aussi adopter des mesures cohérentes en termes de voiries, de stationnement, de priorité aux transports en commun, ce qu'elles tardent à réaliser.

17 BOURTON, W., "Trois lettres : RER comme SOS », Le soir, 22 janvier 1992, p. 20

18 Document du Conseil de la RBC, Bulletin des interpellations et questions orales et d'actualités, Commission de l'infrastructure, chargée des travaux publics et des communications, 18 mars 1998, BIQ (1997-1998) N¹9, p. 8.

19 STRATEC, Evaluation et optimisation des mesures d'accompagnement du RER desservant l'agglomération centre sur la Région de Bruxelles-Capitale, rapport final de la tranche 1 et rapport final de la tranche 2, octobre 2002 et octobre 2003, pour le service fédéral mobilité et transport.

20 Ibid., p. 92 ;. p. 55. 


\section{Le RER : tentatives de régulation politique}

\subsection{Mise à l'agenda, coalitions et expertise (1989-1998)}

12. Ces antagonismes structurants perdureront tout au long de la socio-histoire du RER. Plusieurs phases peuvent être circonscrites dans l'élaboration et la mise en œuvre de ce projet. La première décennie de 1989 à 1999 est caractérisée, d'une part, par le volontarisme des acteurs politiques bruxellois qui n'auront de cesse de visibiliser le projet, de rallier d'autres acteurs à la cause et, d'autre part, par l'importance de la construction de l'expertise autour du dossier. En effet, le ministre bruxellois des transports publics est à l'initiative, en 1991, d'une "Task Force pour l'amélioration de la desserte ferroviaire de Bruxelles " 21 , rassemblant les différentes parties et qui a pour objectif de plancher d'abord sur " la revalorisation de la desserte de la ligne 26 " et ensuite de " dresser l'inventaire de dossiers communs à la SNCB et à la STIB en vue notamment de favoriser la réalisation d'un réseau RER „22. La stratégie du cabinet de Jean-Louis Thys, comme celle des ministres bruxellois qui lui succèderont au chevet du
RER, semble être, d'impliquer les autres acteurs, à l'intérieur mais aussi (et surtout) à l'extérieur de la Région afin que le RER n'apparaisse pas comme une demande purement bruxelloise. En juin 1992, la première table ronde sur le RER est organisée. Elle s'intitule - non sans une certaine ironie de l'histoire - "Le RER à toute vitesse »23. Au cours de cette conférence, les autres acteurs politiques, comme la Flandre, se montrent réticents (voir supra). La création de cette Task Force aboutit toutefois à la création du "Syndicat d'études pour le RER » en juin 1993. L'initiative revient encore une fois à la RBC ${ }^{24}$. La mission de ce syndicat d'études était de proposer un réseau, en donnant priorité à l'infrastructure existante, en programmant les futurs investissements, en précisant le matériel roulant à acquérir, les coûts d'investissement et d'exploitation. II fera appel à un bureau d'études français, SOFRETU, spécialisé dans la conception de réseaux de transport public. Entouré d'autres bureaux sous-traitants, ce syndicat d'étude remet un premier rapport en 1995, souvent dénommé « étude Sofretu » 25

${ }^{21}$ Document du Conseil de la RBC, Rapport fait au nom de la commission de l'infrastructure, chargée des travaux publics et des communications. Réseau Express Régional (RER), Bruxelles, 8 mai 1996, document A85/1 95-96, annexe 1 (Synthèse du projet de RER), p. 34.

22 Conseil de la RBC, compte-rendu intégral, séance plénière du jeudi 13 juin 1991.

23 "Le RER à toute vitesse ", Actes de la table ronde du 5 juin 1992, Bruxelles.

${ }^{24}$ REBUFFAT, J., " La Région paie 35 millions pour une étude sur le futur RER », Le Soir, 3 avril 1993, p. 30

${ }^{25}$ COMITE DE PILOTAGE CONVENTION RER du 04/04/2003 (MB 01/03/2006), Rapport annuel 2006-2007. 
13. Toujours dans une perspective de construction de configurations d'acteurs favorables, une deuxième table ronde est organisée le 12 mars 1996 et rassemble 300 personnes ${ }^{26}$. A partir de cette période, l'appui des deux autres Régions au projet RER semble se dessiner selon certains ${ }^{27}$ : «Un projet de réseau a ainsi été présenté [...] où il a fait l'objet d'un large consensus $2^{28}$. Pour d'autres, comme le Ministre fédéral Michel Daerden, les approches divergent et démontrent le caractère hautement " communautaire $»^{29} \mathrm{du}$ dossier, qu'il ne faut pas présenter comme " un acquis irréversible ${ }^{30}$. De fait, le plan décennal de la SNCB ${ }^{31}$, finalement approuvé en juillet $1996^{32}$, ne reprend pas la réalisation du RER comme une priorité. Le syndicat d'études pour le RER remet un second rapport en janvier 1998, son " rapport final " présentant un projet de RER qui constitue "un réseau articulé principalement autour de la jonction $^{33}$ ». Le projet est dénoncé par certains politiques bruxellois, notamment parce qu'il abandonne la ligne 28 et qu'il ne tient pas compte des recommandations du Conseil de la RBC adoptées en mai 1996. Entre-temps, la SNCB adopte son nouveau plan de transport qui entrera en vigueur en mai 1998. Ce plan, s'il gâte les navetteurs longue distance, oublie " les banlieusards, soit les liaisons à moins de 30 kilomètres autour de Bruxelles. La nouvelle offre ne rencontre pas fondamentalement l'idée du RER [...]”34.
14. Pour les politiques bruxellois, le rapport final du syndicat d'étude doit faire l'objet d'un accord de coopération entre les trois Régions et le fédéral. Si la RBC a manifestement pris des contacts en ce sens ${ }^{35}$, le projet RER entre dans une phase de latence. II faudra plus d'un an pour que la Conférence Interministérielle des Communications et de l'Infrastructure $(\mathrm{ClCl})$ se prononce, en mars 1999, en faveur de la réalisation du RER et de la création d'un groupe de travail, dénommé « groupe RER de haut niveau » mandaté pour étudier (à nouveau) les conditions de réalisation du RER et pour mettre au point un projet d'accord de coopération entre le pouvoir fédéral et les trois Régions.

${ }^{26}$ Document du Conseil de la RBC, Rapport fait au nom de la commission de l'infrastructure, chargée des travaux publics et des communications. Réseau Express Régional (RER), Bruxelles, 8 mai 1996, document A85/1 95-96.

27 Ibid., exposé du ministre Hervé HASQUIN, p. 4.

28 Document du Conseil de la Région de Bruxelles-Capitale, Bulletin des interpellations et questions orales et d'actualités, Commission de l'infrastructure, chargée des travaux publics et des communications, 18 mars 1998, BIQ (1997-1998) Nº19, p. 3

${ }^{29}$ Chambre des représentants de Belgique, Commission de l'Infrastructure, des Communications et des Entreprises Publiques, 20 mars 1996, C105, p. 4.

30 Ministre Daerden, ibid., p. 21

${ }^{31}$ Plan décennal 1996-2005 pour le transport de demain : STAR 21, SNCB, 1996.

32 Troisième avenant au premier contrat de gestion de la SNCB. Publié au Moniteur le 29 octobre 1996.

33 Document du Conseil de la RBC, Bulletin des interpellations et questions orales et d'actualités, Commission de l'infrastructure, chargée des travaux publics et des communications, 18 mars 1998, BIQ (1997-1998) N¹9

34 TELLIER, D., " Le SP appuie le plan Transport SNCB. Le RER n'est pas l'affaire des Bruxellois ", Le Soir, 1'r août 1997, p. 4.

35 Document du Conseil de la RBC, Bulletin des interpellations et questions orales et d'actualités, Commission de l'infrastructure, chargée des travaux publics et des communications, 18 mars 1998, BIQ (1997-1998) Nº19, p. 7 


\subsection{L'institutionnalisation et le début de la mise en oeuvre (1999-2008)}

15. La décennie suivante est davantage une phase décisionnelle importante qui institutionnalise, malgré plusieurs blocages, le projet et qui va également déboucher sur une mise en place, au moins partielle, des infrastructures nécessaires à sa réalisation. Suite aux élections législatives de juin 1999, la déclaration gouvernementale évoque la nécessité de créer le RER ${ }^{36}$. En 2000, une nouvelle conférence interministérielle relance les travaux du «Groupe RER de haut niveau » et charge celui-cl de la mise au point d'un projet et d'un accord de coopération ${ }^{37}$. A la fin de cette même année, le fonds RER est créé pour couvrir une partie des investissements en infrastructures ${ }^{38}$. Le fédéral travaille à la production d'une note RER et propose un projet de future convention RER à négocier avec les Régions. L'avis du CA de la SNCB est cependant requis et il faudra attendre mars 2001 pour que celle-ci exprime un avis favorable concernant le projet RER, inscrit budgétairement dans le plan décennal d'investissement 2001-2012. La SNCB avance cependant à reculons, claironnant souvent qu'elle n'a pas ou plus les fonds pour investir dans ce projet, ce qui provoque un «bras de fer » avec le monde politique, qui dénonce le « gel » du projet ${ }^{39}$. Les acteurs politiques bruxellois, toujours très proactifs sur le dossier, s'inquiètent également de ne pas voir arriver cet accord de coopération qui, finalement prend la forme de la convention du 4 avril 2003 visant à mettre en œuvre le programme du RER de, vers, dans et autour de Bruxelles, et établie par l'Etat fédéral et les trois Régions ${ }^{40}$.

16. Le contenu du projet de RER pour Bruxelles peut être résumé en trois points : la réalisation d'un certain nombre d'infrastructures ferroviaires (mises à quatre voies de plusieurs lignes, aménagement et création de haltes, etc.) et routières (aménagement de liaisons par bus) ; la mise au point de modalités d'exploitation de cette infrastructure (dont le schéma d'exploitation et l'achat de matériel roulant) et la mise en place de " mesures d'accompagnement ", définies dans la Convention de 2003 comme " toute action qui a pour objectif de favoriser l'utilisation des transports en commun dans la zone RER » (la politique de stationnement et la construction de parking, la hiérarchisation des voiries en ville, l'amélioration des conditions d'intermodalité, etc.). La convention crée également un certain nombre d'organes de coordination « ad hoc » pour assurer les négociations entre les parties. A côté du Comité Exécutif des Ministres de la Mobilité (CEMM), composé des quatre ministres ayant la mobilité dans leurs attributions, la convention institue le "Comité de pilotage RER », composé des différentes administrations et des sociétés de transport, ainsi que "le Groupe opérationnel », un groupe plus technique associant uniquement des représentants des quatre sociétés de transport en commun.

17. Chargé du suivi général de la convention, le Comité de pilotage est un groupe central dans la conduite des négociations. Entré en fonction à partir d'avril 2006, il a notamment été chargé du suivi d'une étude, prévue à l'article 13 de la convention, qui porte sur les besoins de déplacements, et sur les demandes de fonctionnalité en matière de fréquence, amplitude et points d'arrêts, c'est-à-dire le « schéma d'exploitation » du RER. Cette étude est confiée, début 2008, à une association de bureaux d'études et fait l'objet de discussions au fur et à mesure de son avancement au sein du Comité de pilotage. Le rapport de cette étude, datant de juin 2009, avance un scénario intermédiaire de mise en place du RER pour 2015, mais il propose également un scénario pour 2020 et un autre pour 2030. Ces scénarios n'ont pas été étudiés aussi intensément du point de vue technique mais ont l'avantage politique de rencontrer davantage les revendications concurrentes des Régions. Si le rapport d'activité des bureaux d'étude a été approu-

${ }^{36}$ Chambre des représentants de Belgique, Annales, séance plénière du 14/07/1999, p. 48.

37 COMITE DE PILOTAGE CONVENTION RER du 04/04/2003, Rapport annuel 2006-2007, p. 6

38 Ibid.

39 Voir par exemple : Demonty, B., Dewez, A., "Le patron de la SNCB affirme qu'il n'a pas de moyens pour entreprendre les travaux RER : bras de fer Vinck-Durant ", Le Soir, 8 février 2003, p. 33 ; Demonty, B., "La ministre des transports n'est toujours pas satisfaite. Un peu d'argent pour le RER », Le Soir, 15 février 2003, p. 31.

40 Convention du 4 avril 2003 visant à mettre en œuvre le programme du Réseau express régional de, vers, dans et autour de Bruxelles. Cette convention a fait l'objet d'une loi, adoptée le 17 juin 2005 et publiée au Moniteur belge, le $1^{\text {er }}$ mars 2006. 


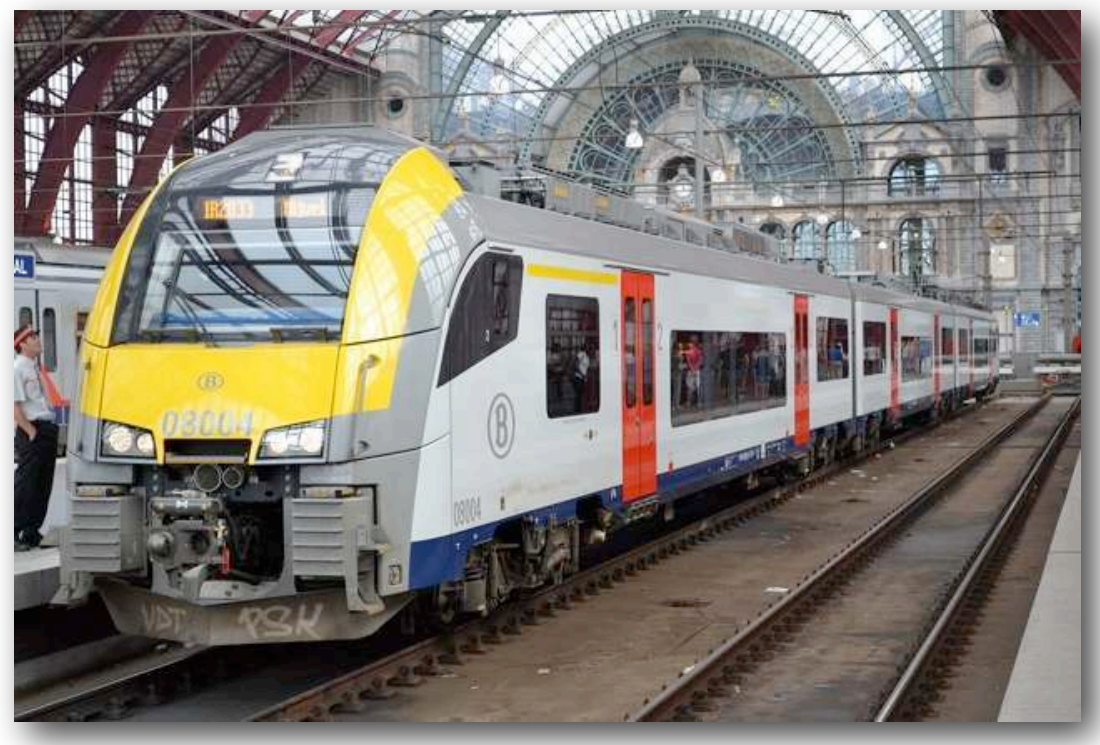

Figure 1. Rame Siemens Desiro ML de la SNCB, déjà en service comme train IR, vue ici en gare d'Anvers central. Source : photo Alfenaar via Flickr/Wikimedia, Creative Commons - certains droits réservés.

vé afin de clôturer le marché, le contenu du rapport n'a fait l'objet, à ce jour, d'aucune acceptation officielle, que ce soit par le Comité de pilotage, les Régions, le Fédéral ou la SNCB.

18. Le projet RER se matérialise à partir de 2004 avec le début (de Schuman à Watermael, par exemple) des premiers travaux d'infrastructure, travaux qui ont nécessité la réalisation d'études d'incidences et l'obtention, non sans mal, de certificats et de permis d'urbanisme. Le matériel roulant a lui aussi fait l'objet d'un contrat en avril 2008, avec Siemens, pour l'obtention de 305 rames Desiro ML dont 95 devraient servir au RER. Ce matériel n'est donc pas spécifiquement dédié au RER, suscitant de nombreuses critiques y voyant une stratégie de l'opérateur de transport pour tirer profit de cette acquisition à destination de son réseau « classique ». Malgré ces avancées, le projet RER entre à nouveau dans un frigo institutionnel.

41 « RER : Un retard de trois ans suite à l'arrêt du Conseil d'Etat ? ", Le Soir, 19 février 2010.

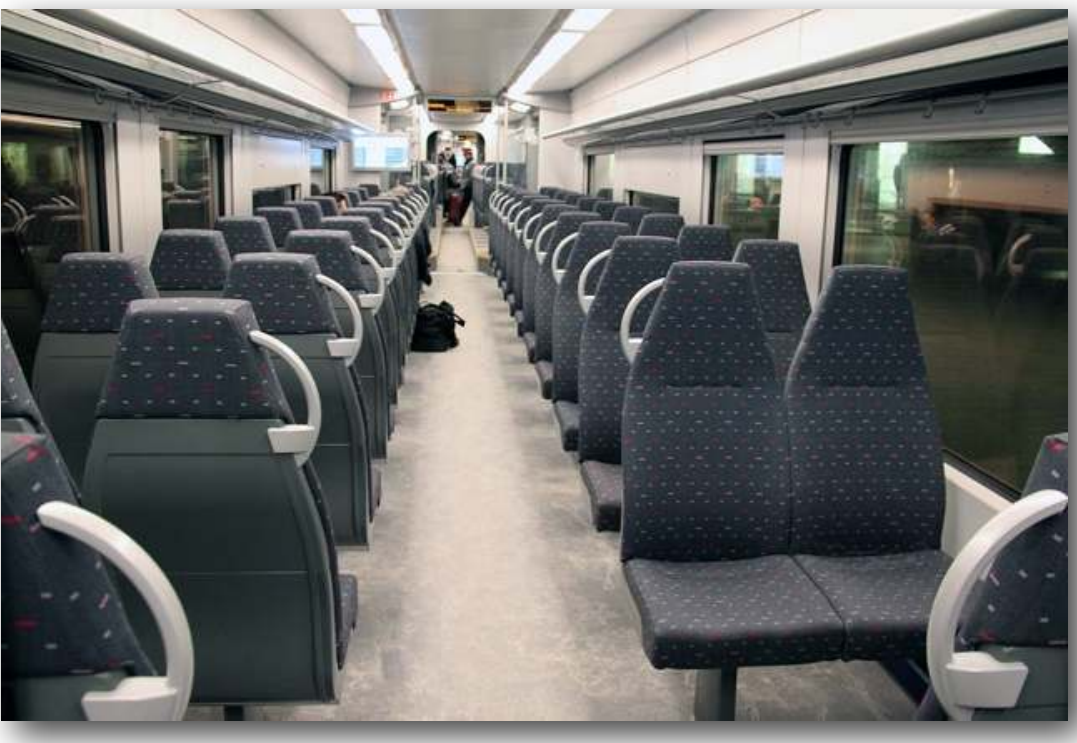

Figure 2. Intérieur (seconde classe) d'une rame Siemens Desiro ML de la SNCB déjà en service. Noter la densité de l'aménagement et l'absence de tablette. Source : photo Maurits90 via Wikimedia, Creative Commons - certains droits réservés.

\subsection{Blocages et alternatives (2009 - )}

19. Depuis 2009, en effet, de nombreux obstacles se dressent sur la route du RER. L'approbation du schéma d'exploitation proposé par l'étude dite « article 13 », a été bloquée par les élections régionales, la mise en place de nouveaux cabinets, puis par la plus importante crise institutionnelle qu'ait connue la Belgique et l'absence subséquente d'un gouvernement fédéral. En 2010, Infrabel faisait également part du retard dans la mise en service du RER en raison de la saga juridique liée à l'obtention des permis à Linkebeek : au lieu de 2016 pour la mise en service, Infrabel évoquait 2019 ou $2020^{41}$. En 2012, la date butoir était estimée cette fois à 2022 tou- 
jours pour les mêmes raisons. Si l'on a pu penser que le RER était " sorti de son hibernation" en mai 2012, suite à une première rencontre des ministres de la mobilité sous l'égide du nouveau Secrétaire fédéral à la mobilité, aucune approbation formelle du schéma d'exploitation n'a eu lieu. Le groupe de pilotage n'avait pu, lui non plus, y parvenir en raison d'oppositions politiques quant aux choix posés par rapport aux usages privilégiés du RER mais aussi d'oppositions plus " techniques " de la SNCB jugeant le scénario intermédiaire 2015 à présent irréalisable, si on tient compte de la situation actuelle d'utilisation (et non de la situation de 2008) et de l'entièreté du réseau (et non d'un modèle qui, selon elle, ne prendrait pas en compte toutes les subtilités du réseau). Sans manifestement trancher ces conflits, la CEMM s'est néanmoins fixé des objectifs communs, rappelant la nécessité d'arriver à une mise en service du RER en 2018, sur base du scénario intermédiaire 2015. Conscients des difficultés de coordination, les acteurs politiques semblent chercher d'autres manières de s'accorder que ce soit via la création d'une " communauté métropolitaine " [Van Wynsberghe, 2013] au sein de laquelle se tiendrait la concertation entre les trois Régions relative, notamment, à la mobilité - mais le RER n'en fera pas explicitement partie - ou encore dans l'évocation d'une nouvelle " filiale dans laquelle les trois Régions et l'Etat fédéral seront représentés ", créée au sein de la SNCB pour " gérer l'exploitation » du RER [Déclaration gouvernementale d'Elio Di Rupo, décembre 2011, p. 27]. Plus récemment, les discussions sur le nouveau plan décennal 2013-2025 de la SNCB sont venues à nouveau mettre en doute l'arrivée prochaine du RER et rappeler l'importance du facteur budgétaire et le manque de détermination de l'entreprise publique sur ce projet. Les montants des investissements en infrastructure ont été réduits, des travaux ont été reportés sine die et d'autres ne sont pas évoqués alors même que la CEMM avait entériné leurs réalisa- tions. A cette occasion, la SNCB affirme aussi qu'une partie des objectifs du RER sont en fait déjà rencontrés par le plan IC/IR, dévoyant de fait le projet qui apportait une offre supplémentaire par rapport à l'existant. Les critiques face à ce plan décennal, dont celles du Gouvernement bruxellois, affluent mais les CEO de la SNCB se défendent : dans un contexte de rareté budgétaire, la priorité doit aller à la sécurisation du rail. Par ailleurs, disent-ils, le rail n'a pas vocation à être un « taxi »42

20. Dans ce contexte tendu, des projets potentiellement alternatifs entrent également dans le débat public. Sans développer ici le projet de réseau de trams "Brabantnet "43 de la société régionale flamande de transport public De Lijn qui vise aussi à désengorger Bruxelles, pensons aux propositions de Jannie Haek ${ }^{44}$, patron de la SNCB-Holding, sans doute inspirées par le projet des écologistes bruxellois de Réseau Express Bruxellois (REB). Ce dernier projet s'appuie sur l'infrastructure existante (dans un premier temps) pour proposer deux lignes dans Bruxelles (de Moensberg vers le centre et de Berchem vers le centre), s'arrêtant dans les gares actuelles, souvent sous-exploitées selon Ecolo/Groen. En utilisant les lignes 26, 25 et 50 essentiellement, le REB conçoit une offre de trains cadencés tous les quarts d'heure et qui relient de nombreuses communes bruxelloises entre elles. Moyennant des investissements relativement limités, le REB est présenté comme pouvant apporter une solution rapide pour les Bruxellois, complémentaire à une offre RER visant davantage les navetteurs. Ce projet est loin d'être anecdotique dans la mesure où il suscite beaucoup de réactions dont celles de différentes communes de Bruxelles qui se sont prononcées sur une motion déposée par les écologistes (voir les cartes des deux phases du projet).

42 Cfr le débat sur le Plan d'Investissement Pluriannuel de la SNCB devant la Commission Infrastructure du Parlement Bruxellois du 22 mai 2013 ; Baele, M., "Le train pour booster la mobilité dans Bruxelles? Impossible, dit la SNCB ", RTBF info, 23 mai 2013. Disponible en ligne : www.rtbf.be/info/regions/detail le-train-pour-booster-la-mobilite-dans-bruxelles-impossible-dit-la-sncb?id=8001244 ${ }^{43}$ Voir http://www.delijn.be/mobiliteitsvisie2020/pegasus vlaamsbrabant/index.htm

${ }^{44}$ Renette, E., « Une alternative ferroviaire au RER » et « Une petite ceinture version rail », Le Soir, 19 avril 2013 ; « RER : la proposition de la SNCB déplait », La Libre, 19 avril 2013. 
Réseau Express Bruxellois (REB) |Brussels Express Netwerk (BEN) Phase 1 Fase 1

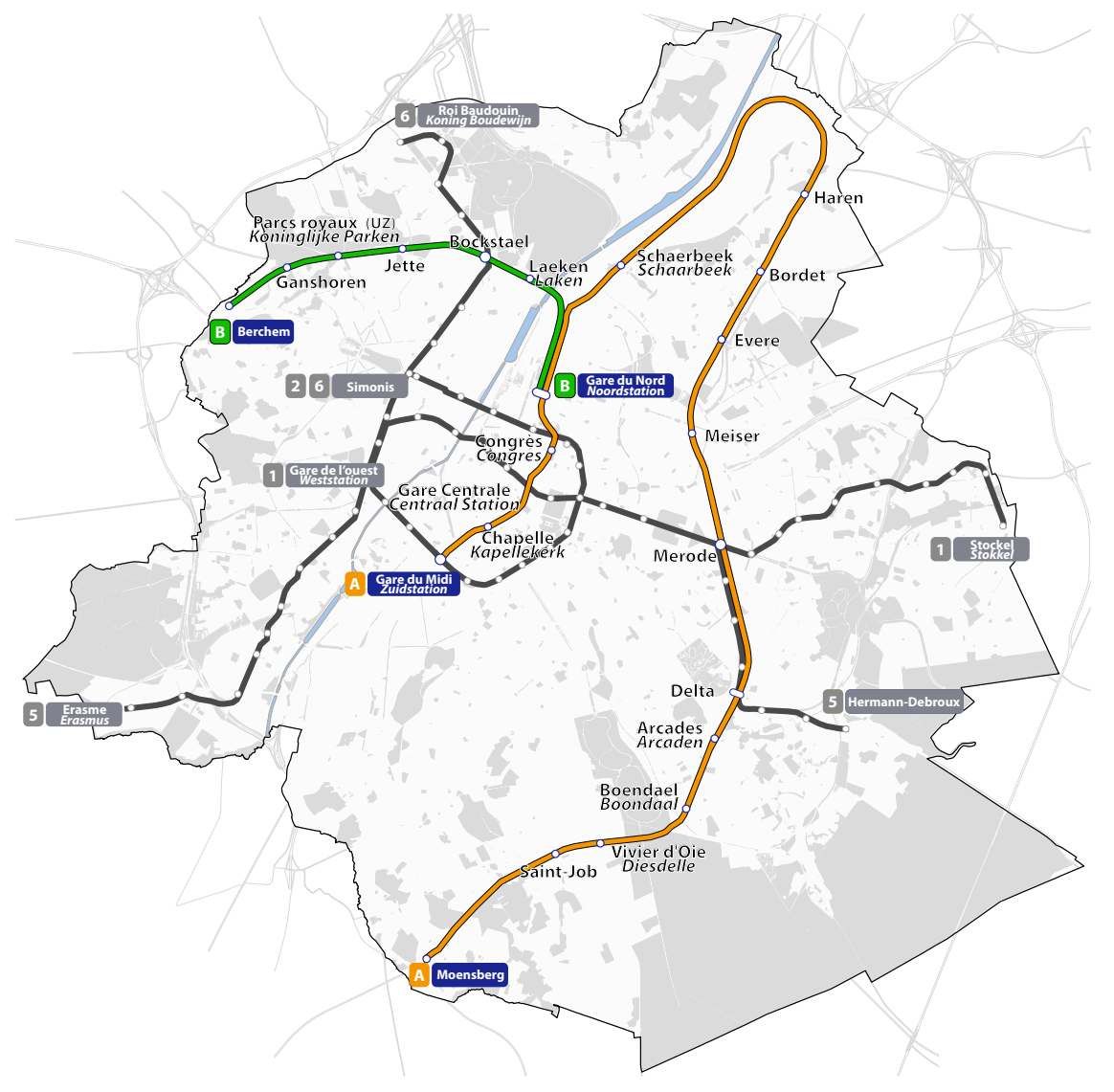

Réseau Express Bruxellois (REB)|Brussels Express Netwerk (BEN) \begin{tabular}{l|l} 
Phase 2 & Fase 2
\end{tabular}

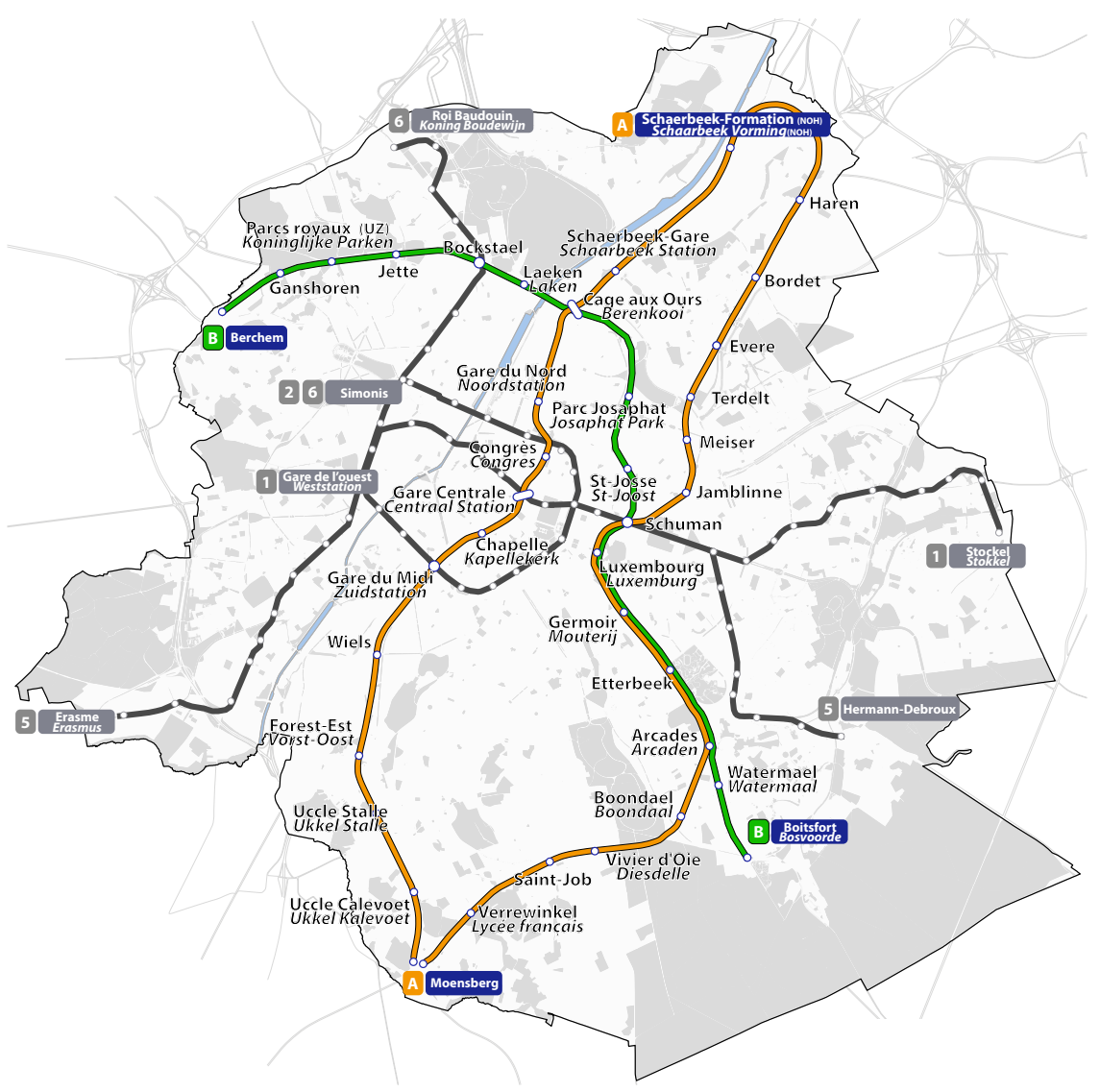

Figures 3 \& 4. Projet de Réseau Express Bruxellois (REB) des écologistes bruxellois. Source : Ecolo-Groen, 2013. 
21. Dans une perspective analogue, quoique plus urbanistique, un processus réflexif et artistique de deux ans, initié par les asbl Recyclart et Congres, avec le Maitre architecte pour la Région bruxelloise et l'Agence de développement territorial, a conduit le 12 décembre 2013 à la présentation d'un "Manifeste Jonction » qui reprend l'idée de deux boucles (dites " papillon " en raison de leur forme) de contournement est et ouest de la Jonction Nord-Midi, favorable au développement d'un RER intra-bruxellois et d'une ville polycentrique ${ }^{45}$ II promeut également une rénovation en surface et en sous-sol de ladite Jonction et envisage une traversée facilitée de Bruxelles par les trains internationaux, le tout dans une perspective de "reprise en main » de l'ensemble de cette problématique par les autorités régionales bruxelloises.

Figure 5. Les deux boucles "papillon », contournement est et ouest de la jonction Nord-Midi. Source : Manifeste Jonction (www.jonction.be), 2013.

45 Lors de leur présentation, les auteurs du Manifeste ont fait explicitement référence au bureau 51N4E qui, le premier, a développé cette idée dans le cadre de l'étude « Bruxelles 2040 », réalisée en 2011 à la demande de la RBC, et qui a fait l'objet, en 2012, d'une exposition au Palais des Beaux-Arts de Bruxelles. 


\section{Conclusion}

22. Le RER est un dossier complexe, marqué par des enjeux sociaux politiques et économiques importants. II apparaît d'abord comme emblématique de l'antagonisme politique, dans un pays marqué par un fédéralisme de dissociation [Delpérée, 2011 ; Delwit et Pilet, 2004], basé sur des stratégies d'attractivité opposées des entités fédérées. Les usages que le RER entend privilégier et les visions de Bruxelles en jeu sont clairement concurrentes et n'apparaissent pas dépassées à ce stade par le projet. En outre, dans la mesure où il implique, au-delà des politiques sectorielles du transport déjà fort complexes en elles-mêmes, de réfléchir à d'autres actions publiques connexes, de développer, donc, des stratégies de désectorialisation, le dossier RER se complique encore. Les réflexions croisées entre usage du rail et préoccupations environnementales poussent en particulier à la coordination des actions entre transport et aménagement du territoire [Gauthier, 2005], mais la volonté politique semble manquer pour adopter des stratégies désectorialisées et cohérentes en la matière.

23. Par ailleurs, le dossier RER est également façonné par différentes dimensions dont les logiques et temporalités s'ajustent difficilement. Les logiques d'expertise des bureaux d'étude et le monde des techniciens et des ingénieurs des entreprises de transport ne rencontrent pas forcément les temporalités du politique et ses logiques de décision, souvent inscrites dans des processus qui ont également leurs accélérations et ralentissements. L'opérateur de transport SNCB a par ailleurs ses propres stratégies, privilégiant incontestablement ce qu'il considère comme son " core-business » en tant qu'entreprise « fédérale ». S'ajoutent à cela également les logiques sociétales en termes de déplacements et de localisations résidentielles et autres, qui sont bien évidemment liées aux politiques menées et aux infrastructures de transport, mais ont également leurs propres dynamiques. Finalement, ce dossier témoigne encore d'un compromis, typiquement belge peut-être, puisque les questions liées aux travaux, à l'infrastructure lourde et au matériel roulant ont été solutionnées, au moins en partie, avant de régler les questions plus sensibles de l'offre concrète de transport ainsi que des politiques annexes qui doivent en réguler l'usage. Comme s'il était donc logique de construire une machine avant de savoir exactement à quoi elle va servir. Sans doute faut-il y voir la force ou, en tout cas, «le caractère opératoire du quiproquo » [Offner, 2012]. Les oppositions ne sont pas levées, les incertitudes perdurent, précisément parce qu'elles permettent à chacun des acteurs d'espérer voir ses propres visions triompher un jour. 


\section{Bibliographie}

AUSSEMS, M., 2009. D'une politique des transports à une politique de mobilité globale ? In : BEAUFAYS, Jean, MATAGNE, Geoffroy, La Belgique en mutation. Systèmes politiques et politiques publiques (1968-2008). Bruxelles : Bruylant. pp. 285-311.

BOON, Françoise, GAYDA, Sylvie, 2000. Quelques impacts du futur RER sur la région bruxelloise et sa périphérie. In : 14e Congrès des Economistes Belges de Langue Française, novembre 2000. disponible en ligne :

http://www.stratec.be/sites/default/files/files/IMPACTSRER2-FB.pdf

CASABELLA, Nadia, FRENAY, Patrick, 2009. Regional Planning Choices: Comparing the RER in Brussels (BE) and the Stedenbaan in South-Holland (NL). In : The 4th International Conference of the International Forum on Urbanism. Amsterdam/Delft.

DAMAY, Ludivine, DENIS, Benjamin, DUEZ, Denis, 2011. Savoirs experts et profanes dans la construction des problèmes publics. Bruxelles : Publications des Facultés universitaires Saint-Louis.

DE KEERSMAECKER, Marie-Laurence, 2005. Bruxelles face à sa mobilité. In : DECLEVE, Bernard, DE RIJCK, Kim (dir.), Usages et paysages de la ville en mouvement. Bruxelles et la ligne 161, LouvainLa-Neuve : Presses universitaires de Louvain. pp. 95-110.

DELPEREE, Francis, 2011. La Belgique existe-t-elle ? In : Pouvoirs. n 136, pp. 9-19

DELWIT, Pascal, PILET, Jean-Benoit, 2004. Fédéralisme, institutions et vie politique. Stabilité, instabilité et retour. In : COENEN, Marie-Thérèse, GOVAERT, Serge, HEINEN, Jean (Ed.), L'Etat de la Belgique. 1989-2004 : Quinze années à la charnière du siècle. Bruxelles : De Boeck \& Larcier. pp. 43-79.

DESSOUROUX, Christian, 2008. Le poids des héritages. In : Transports urbains. $\mathrm{n}^{\circ} 114$, pp. 4-7.

DOBRUSZKES, Frédéric, 2008. Un cadre peu propice à l'utilisation des transports collectifs. In : Transports urbains. $n^{\circ} 114$, pp. 8-15.
FRENAY, Patrick, 2009. Pour un projet de développement territorial associé au RER bruxellois. Essai de mise en évidence des facteurs déterminants fondamentaux. In : Brussels Studies. n³1, www.brusselsstudies.be.

GAUTHIER, M., 2005. La planification des transports et le développement durable à Montréal : quelles procédures de débat public pour quelles solutions intégrées ? In : FLUX. n60-61, avril-septembre, pp.50-63.

HUBERT, Michel, 2008. L'Expo 58 et le «tout à l'automobile ». In : Brussels Studies. n²2, www.brusselsstudies.be.

HUBERT, Michel, DOBRUSZKES, Frédéric, MACHARIS, Cathy, 2008 Etats généraux de Bruxelles. La mobilité de, à, vers et autour de Bruxelles. In : Brussels Studies, note de synthèse $n^{\circ} 1$, www.brusselsstudies.be.

MONTULET Bertrand, HUYNEN Philippe, HUBERT Michel, 2008. Belgium - A Society of Commuters. In : SCHNEIDER N.F., MEIL G. (dir.). Mobile Living Across Europe I. Relevance and Diversity of JobRelated Mobility in Six European Countries. Barbara Budrich Publishers: Opladen \& Farmington Hills. pp. 269-304.

NOIRIEL, Gérard, 2006. Introduction à la socio-histoire. Paris : La Découverte.

OFFNER, Jean-Marc, 2012. L'action publique entre raisons et déraisons. In : Revue Urbanisme. n`385, juillet-août, pp. 27-28.

SCHOONBRODT, René, 2007. Vouloir et dire la ville. Bruxelles. AAM éditions.

TELLIER, Céline, 2012. Corps technique et techniques du corps. Sociologie des ingénieurs du souterrain bruxellois (1950-2010). Thèse de doctorat. Bruxelles : Université Libre de Bruxelles.

VAN MEERTEN, Michelangelo, VERBEURGT, Greta, VAN DER HERTEN, Bart, 2002. Un tunnel sous Bruxelles. Les 50 ans de la jonction Nord-Midi. Bruxelles : Editions Racine.

VAN WYNSBERGHE, Caroline, 2013. Bruxelles et Washington : deux capitales fédérales enclavées, deux expériences métropolitaines comparables ? In : Brussels Studies, n66, www.brusselsstudies.be. 


\section{Soutien financier}

Brussels Studies est publié avec le soutien de :
$\begin{array}{ll}\text { INNOVIRIS } & \text { Innoviris, l'Institut Bruxellois pour la } \\ & \text { Recherche et l'Innovation }\end{array}$

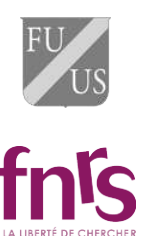
Fondation Universitaire

\section{fnls}
Fonds ISDT Wernaers pour la vulgarisation scientifique - FNRS

\section{Pour citer ce texte}

Ludivine DAMAY, 2014. Un RER à Bruxelles ? Socio-histoire des rivalités et des régulations politiques (1989-2013). In : Brussels Studies, Numéro 74, 17 février 2014, www.brusselsstudies.be.

\section{Liens}

D'autres versions de ce texte sont disponibles ePub FR: http://tinyurl.com/BRUS74FREPUB ePub NL : http://tinyurl.com/BRUS74NLEPUB ePub EN : http://tinyurl.com/BRUS74ENEPUB pdf FR : http://tinyurl.com/BRUS74FRPDF pdf NL : http://tinyurl.com/BRUS74NLPDF pdf EN : http://tinyurl.com/BRUS74ENPDF 